Carnets de géographes

GÉOGRAPHES

\title{
Performances touristiques et production des identités spatiales individuelles à Amsterdam
}

Amandine Chapuis

\section{OpenEdition}

Journals

Édition électronique

URL : http://journals.openedition.org/cdg/889

DOI : $10.4000 /$ cdg.889

ISSN : 2107-7266

Éditeur

UMR 245 - CESSMA

\section{Référence électronique}

Amandine Chapuis, «Performances touristiques et production des identités spatiales individuelles à Amsterdam », Carnets de géographes [En ligne], 6 | 2013, mis en ligne le 01 septembre 2013, consulté le 23 septembre 2020. URL : http://journals.openedition.org/cdg/889 ; DOI : https://doi.org/10.4000/ cdg.889

\section{(c) (i) (3)}

La revue Carnets de géographes est mise à disposition selon les termes de la Licence Creative Commons Attribution - Pas d'Utilisation Commerciale - Pas de Modification 4.0 International. 


\title{
PERFORMANCES TOURISTIQUES ET PRODUCTION DES IDENTITÉS SPATIALES INDIVIDUELLES À AMSTERDAM
}

\author{
AMANDINE CHAPUIS
}

À partir d'une analyse des pratiques touristiques à Amsterdam, je questionne dans cette thèse le rôle du tourisme dans la construction de la dimension spatiale des identités individuelles. Tandis que les pratiques récursives des environnements familiers sont désormais bien prises en charge par les études urbaines, les pratiques liées au tourisme et les espaces vécus qu'elles dessinent restent encore peu abordés en géographie. Pourtant, le tourisme est un phénomène ancien, en croissance constante. Un des principaux vecteurs de la mondialisation culturelle, il touche une part de plus en plus nombreuse de la population mondiale, investit et subvertit de plus en plus d'espaces urbains.

Je propose une géographie intime de la pratique touristique, en plaçant l'échelle de l'individu au cœur de la réflexion. Il s'agit de prendre au sérieux des expériences personnelles, tout en montrant comment elles participent aux multiples processus d'identification qui sont à l'œuvre chez les individus. Je mobilise la notion de "performance », que j'emprunte notamment à Judith Butler, pour appréhender cette relation entre pratiques, espace et processus d'identification. Définissant les performances touristiques comme les pratiques in situ d'un individu, en ce qu'elles incarnent des normes socio-culturelles, mais aussi en ce qu'elles participent en même temps à leur reproduction et/ou à leur subversion, j'ai cherché à comprendre comment, à la faveur de leurs performances touristiques d'Amsterdam, les touristes mettent en œuvre et produisent à la fois la dimension spatiale de leurs identités.

Amsterdam est un lieu touristique majeur en Europe et un espace urbain très divers. Elle est investie et appropriée par des pratiques touristiques qui interagissent avec les dynamiques urbaines contemporaines (patrimonialisation, esthétisation, normalisation des espaces publics). L'analyse de l'évolution du projet urbain m'a permis de montrer que le tourisme est devenu un principe générateur de la fabrique de la ville. J'apporte une lecture problématisée de la manière dont une métropole a produit et gère une rente de monopole touristique, marquée par une tension importante entre différents imaginaires, différents types de pratiques, des plus initiatiques et festives, depuis les mouvements hippies des années soixante-dix, aux plus légitimes culturellement, à travers l'exploitation marchande de l'héritage du Siècle d'Or hollandais. La production de l'espace public, en particulier central, en tant que vitrine mondiale de consommation, renvoie directement à cette dichotomie, définissant progressivement des publics désirés et indésirables, ce qui permet de nuancer une vision idyllique et réductrice d'Amsterdam comme « ville juste ».

Cependant, elle n'est pas un simple cadre de l'expérience. La pratique touristique déploie des spatialités propres, une géographie intime de la ville. Le tourisme mobilise des compétences, des habitus et des représentations spatiales, qui sont reformulés, questionnés, renforcés et parfois subvertis par l'expérience in situ. J'analyse l'imbrication des ressources spatiales et 
des ressources sociales dans la performance touristique. Je mets aussi en évidence les mécanismes par lesquels les individus mettent en cohérence les différents lieux qui constituent leurs espaces de vie, dans le cadre d'une mondialisation culturelle où la mobilité est non seulement une réalité croissante, mais aussi une injonction sociale grandissante. Je montre que les espaces de vie des personnes que j'ai interrogées sont à la fois polytopiques (c'est-à-dire que plusieurs lieux sont investis de sens, et pas seulement le lieu de résidence), réticulaires (car ces lieux sont reliés les uns aux autres, comparés, hiérarchisés) et multiscalaires (car les échelles de référence de la performance les mettent en regard, indépendamment de la logique de l'emboîtement des échelles). La pratique touristique participe ainsi au processus performatif de construction des citadinités individuelles.

Amsterdam, associée à un imaginaire touristique lié à l'initiation (par le sexe, l'alcool, la drogue), constitue un terrain privilégié pour questionner les normes spatiales incorporées par les individus. La question des rapports sociaux de sexe et de l'expérience genrée de la ville est explicitement posée en lien avec l'analyse du fonctionnement et des lectures touristiques du Quartier Rouge. Les lieux apparaissent alors comme des supports d'identification non seulement individuels mais aussi collectifs. Je mets en lumière la manière dont Amsterdam parle à différents groupes transnationaux, par exemple la communauté gay, mais aussi les mouvements chrétiens, qui l'investissent massivement. Je montre comment la performance d'Amsterdam est un moment privilégié d'affirmation de sa propre appartenance à ces groupes à l'échelle individuelle.

Le protocole méthodologique développé est essentiellement constitué d'approches qualitatives. En effet, à travers l'exploitation des données statistiques produites par l'office du tourisme local, je montre les limites heuristiques d'une approche quantitative; à l'inverse, l'enquête ethnographique s'est avérée extrêmement féconde. J'ai placé le recueil des récits touristiques in situ au cœur du protocole d'enquête. L'observation participante, principalement en auberges de jeunesse, occupe une place centrale dans la thèse, car interroger la spécificité de la jeunesse dans l'expérience touristique, c'est se donner les moyens de plonger au cœur des problématiques identitaires, qui se construisent tout particulièrement à ce moment biographique. En amont, l'observation des arts de faire des touristes avec les lieux a permis de caractériser précisément les modalités matérielles de la performance touristique d'Amsterdam. En aval, des entretiens approfondis de touristes ayant visité la ville parfois récemment, parfois plusieurs années auparavant ont permis de questionner des profils plus spécifiques et la dimension biographique du voyage à Amsterdam.

Fiche informative

\section{Discipline}

Géographie

\section{Directeur}

Rémy Knafou

\section{Université}

Paris 1 Panthéon Sorbonne

Membres du jury de thèse, soutenue le 30 novembre 2012

- Jean-François Staszak, Professeur à l'Université de Genève, Président du jury 
- Claire Hancock, Maîtresse de Conférences, Université Paris Est Créteil Val-de-Marne, Rapporteure

- Michel Lussault, Professeur, École Normale Supérieure de Lyon, Rapporteur

- Maria Gravari-Barbas, Professeure, Université Paris 1 Panthéon Sorbonne, Examinatrice

- Claudio Minca, Professeur, Université de Wageningen, Examinateur

- Rémy Knafou, Professeur émérite, Université Paris 1 Panthéon Sorbonne, Directeur

\section{Situation professionnelle actuelle}

ATER à l'Université Paris Est Créteil Val-de-Marne, rattachée au laboratoire EIREST, Université Paris 1 Panthéon Sorbonne

\section{Courriel de l'auteure}

amandine.chapuis@gmail.com 